\title{
Distributed Adaptation Decision-Taking Framework and Scalable Video Coding Tunneling for Edge and In-Network Media Adaptation
}

\author{
Michael Grafl, Christian Timmerer, Markus Waltl \\ Alpen-Adria-Universität \\ Klagenfurt, Austria \\ \{firstname.lastname\}@itec.aau.at
}

\author{
George Xilouris, Nikolaos Zotos \\ NCSR Demokritos \\ Athens, Greece \\ \{xilouris, nzotos\}@iiit.demokritos.gr
}

\author{
Daniele Renzi, Stefano Battista \\ bSoft Ltd. \\ Macerata, Italy \\ \{daniele, bautz\}@bsoft.net
}

\author{
Alex Chernilov \\ Optibase \\ Herzlia, Israel \\ Alexc@optibase.com
}

\begin{abstract}
Existing and future media ecosystems need to cope with the ever-increasing heterogeneity of networks, devices, and user characteristics collectively referred to as (usage) context. The key to address this problem is media adaptation to various and dynamically changing contexts in order to provide a service quality that is regarded as satisfactory by the end user. The adaptation can be performed in many ways and at different locations, e.g., at the edge and within the network resulting in a substantial number of issues to be integrated within a media ecosystem. This paper describes research challenges, key innovations, target research outcomes, and achievements so far for edge and in-network media adaptation by introducing the concept of Scalable Video Coding (SVC) tunneling.
\end{abstract}

Keywords-distributed adaptation decision-taking, SVC tunneling, research challenges, in-network adaptation, contentaware networking

\section{INTRODUCTION \& PROBLEM STATEMENT}

The Universal Multimedia Access (UMA) [1] is omnipresent thanks to the evolution of device and network infrastructure technologies and we are now effectively entering the era of Universal Multimedia Experience (UME) [2]. An important aspect towards UMA and UME is the adoption of scalable media coding formats such as Scalable Video Coding (SVC) [3] enabling edge and in-network adaptation. This paper proposes the exploitation of these scalable media formats within the (core) network - with in-network adaptation enabled - in order to optimize the network resources utilization, and at the edge of the network, for the adaptation from/to heterogeneous formats, devices, and platforms. This is achieved by means of overlay networks, where the adaptation is coordinated in a distributed fashion. This innovation approach is referred to as SVC tunneling and the distributed coordination thereof aiming for a better network resource utilization while maintaining a satisfactory Quality of Experience (QoE). The work is conducted as part of the EU FP7 Integrated Project ALICANTE (Media Ecosystem Deployment Through Ubiquitous Content-Aware Network Environments) [4] developing a media ecosystem comprising among others - Home-Box (HB) and Content-Aware Network
(CAN) overlay networks. For an overview of the ALICANTE architecture the reader is referred to [5].

The heterogeneity of devices, platforms, and networks is and most likely will be a constant companion within (future) media (Internet) ecosystems. Thus, we need to provide tools to cope with that heterogeneity in order to support a maximum of use cases while optimizing (network) resource utilization and improving QoE. One such tool is the SVC tunneling approach featuring edge and in-network media adaptation for which research challenges are highlighted in the following.

Distributed adaptation decision-taking framework:

- Where to adapt? At the content source, within the network (with multiple options), at the receiving device, and combinations thereof.

- When to adapt? At request and during the delivery enabling dynamic, adaptive streaming based on the users' context.

- How often to adapt? Too often may increase the risk of flickering, whereas too seldom may result in stalling, both have a considerable impact on the QoE.

- How to adapt? The optimization towards bitrate, resolution, framerate, signal-to-noise ratio (SNR), modality, accessibility, region-of-interest (ROI), etc. results in (too) many possibilities and often depends on the actual content, genre, and application.

Efficient, scalable SVC tunneling and signaling thereof:

- Minimum quality degradation and scalability w.r.t. the number of parallel sessions and acceptable (end-toend) latency. How can transcoding and adaptation steps be organized to minimize impact on QoS and video quality? How many parallel sessions can be supported on network and client equipment?

The impact on the QoS/QoE:

- The QoS/QoE trade-off for the use cases and applications developed in ALICANTE. One example is 


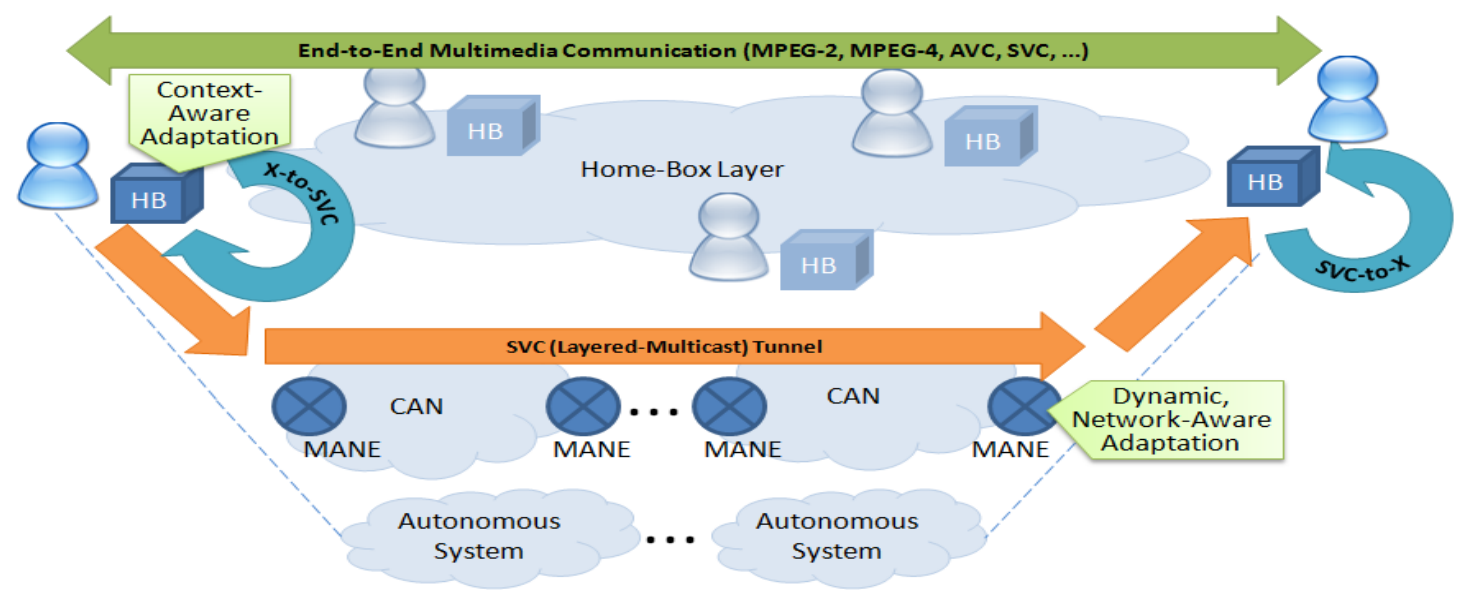

Figure 1. Adaptation Framework Overview.

the trade-off between quality degradation due to transcoding against the QoE gain of dynamic bitrate adaptation.

- Possible mappings of QoS to QoE. Established network QoS parameters (such as packet loss, delay, and jitter) as well as objective video quality are taken into account for estimating the viewing experience.

The adaptation framework and related key innovations are described in Section II. Target research outcomes, our test-bed setup, and scientific results so far are highlighted in Sections III and IV respectively. Section IV also presents test results comparing rate control modes for SVC tunneling. Finally, Section $\mathrm{V}$ concludes the paper and gives an outlook on future work.

\section{THE ALICANTE ADAPTATION FRAMEWORK}

\section{A. Architecture}

The ALICANTE system architecture introduces two new virtual layers, i.e., HB and CAN layers, on top of the existing network infrastructure. This approach brings both contentawareness to the network layer and context-awareness to the user environment.

A full description of the ALICANTE architecture can be found in [5]; this paper rather focuses on the adaptation framework of that architecture. Fig. 1 shows an overview of the ALICANTE adaptation framework. Content delivery in the core network relies on scalable media formats such as SVC. This enables content-aware adaptation according to the network conditions at the CAN layer, i.e., within the MediaAware Network Elements (MANEs).

Home-Boxes are enhanced home gateways with media processing capabilities. They can serve as home media servers, enable users to act as content providers, and keep track of the capabilities of connected terminals. Home-Boxes form a virtual HB layer that enables context-aware adaptation towards end user terminals and user preferences. For example, screen resolution and decoding capabilities are taken into account at content request time. For legacy terminals that do not support SVC, HBs are able to transcode content to non-scalable media formats (e.g., MPEG-2, MPEG-4 AVC). On the server side, corresponding HB layer entities are implemented as software modules.

\section{B. Related Work}

Similar to ALICANTE, several other research projects target media adaptation and content-aware networks. The FP7 Project ENVISION [6] proposes a multi-layered content distribution approach, targeting optimized end-to-end performance and content adaptation during distribution. However, it does not focus on QoE aspects on the client side. Dynamic and distributed adaptation of scalable multimedia content has been proposed by the FP6 Project DANAE [7]. With a focus on the MPEG-21 standard, it pioneered in the area of interoperable adaptation approaches. The FP6 Project ENTHRONE [8] developed a system architecture to cover the entire multimedia distribution chain, focusing on end-to-end QoS performance and network management. These projects tackle important aspects of media-aware adaptation along the delivery path. In the following we discuss several adaptationrelated features of the ALICANTE architecture.

\section{Adaptation Decision-Taking}

Due to multiple locations within the delivery network where content may be subject to adaptation, we propose a distributed Adaptation Decision-Taking Framework (ADTF) that coordinates the local adaptation decisions of modules at the content source, the border to the user (Home-Box), and within the network at MANEs. Local adaptation decisions are taken based on an optimization algorithm, determining the most suitable adaptation for a given content, taking various aspects (e.g., user terminal capabilities, network monitoring results) into account. The various local adaptation decisions have different purposes, depending on the location they are performed in. For example, adaptation decisions in the network focus on dynamic adaptation towards network conditions, while adaptation decisions at the $\mathrm{HB}$ mainly target the capabilities of the user terminal and the QoE. The distribution of adaptation decisions also depends on the streaming mechanism, as, e.g., RTP multicast streaming is handled differently from HTTP streaming. 


\section{SVC Tunneling}

In order to address the problem statement in Section I we propose an SVC (layered-multicast) tunnel, inspired by IPv6 over IPv4 tunnels. That is, within the CAN only scalable media resources - such as SVC - are delivered adopting a layeredmulticast approach [9]. This allows the adaptation of scalable media resources by MANEs [10], implementing the concept of distributed adaptation [11], [12]. At the border to the user (Home-Box), adaptation modules are deployed enabling device-independent access to the SVC-encoded content by providing $\mathrm{X}$-to-SVC and SVC-to-X transcoding/rewriting functions with $X=\{$ MPEG-2, MPEG-4 Part 2, MPEG-4 Part 10 (AVC) etc. $\}$. An advantage of this approach is the reduction of the load on the network (i.e., no duplicates), making it free for (other) data (e.g., more enhancement layers).

First measurements of SVC-based adaptation in an off-theshelf WiFi router [13] have shown evidence to further pursue this kind of approach. More complex adaptation operations that are required to create scalable media resources, such as transcoding of media resources which have higher memory or CPU requirements, will be performed at the edge nodes, i.e., in the HB.

Note that SVC tunneling is also applicable to unicast scenarios due to dynamic SVC-based adaptation, although multicast scenarios bring higher gains in terms of network resource utilization.

\section{E. In-Network Adaptation}

MANEs perform dynamic in-network adaptation to mitigate the effects of network congestion. Each MANE has a local Adaptation Decision-Taking Engine (ADTE) that computes whether to adapt a media stream. The adaptation processes for multicast and unicast streaming have to be considered separately. Multicast streaming deploys RTP multisession transmission (MST) mode, where SVC layers are transmitted over separate RTP sessions and are rearranged by the receiver. Thus, multicast trees for the different SVC layers are built. MANEs realize dynamic adaptation by pruning (or conversely grafting) the multicast tree corresponding to a specific SVC layer.

RTP-based unicast streaming is typically realized via single session transmission (SST) mode, where the entire SVC stream is packed into a single RTP session. In order to perform adaptation, a MANE de-packetizes the RTP stream, analyzes the SVC header, and filters SVC layers accordingly [14]. The RTP re-packetization module updates the sequence number field of the RTP packet headers if needed. Alternatively, unicast streaming could also be realized via MST mode, using separate ports for separate layers.

\section{F. Scalability Considerations}

The proposed techniques act on a per-flow basis, thus, some scalability considerations (in terms of number of concurrent flows) have to be taken into account. Adaptation decision-taking at a MANE has to handle many different flows in parallel, requiring a very lean and efficient implementation of the ADTE. The processing overhead can be controlled by the update frequency of adaptation decisions. For example, the

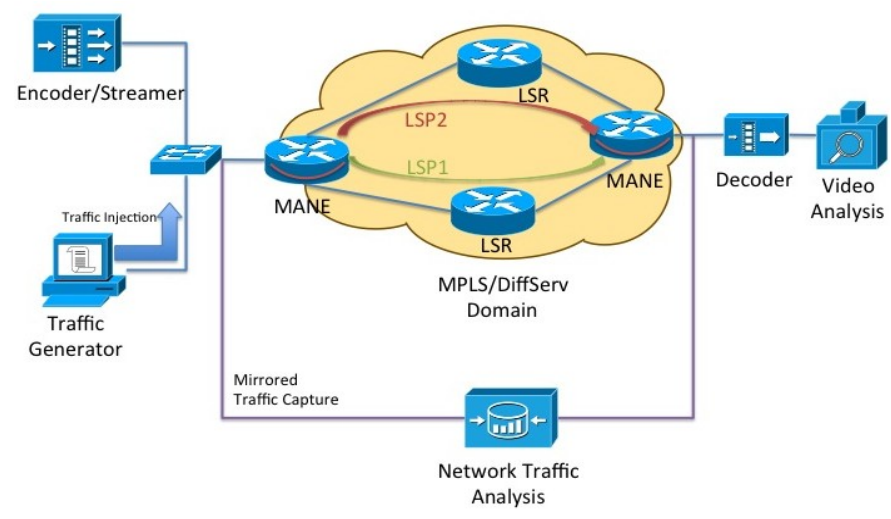

Figure 2. Test-bed setup.

decision to drop an SVC layer shall be triggered immediately when network monitoring indicates congestion, but the decision to add a layer back to the stream can be delayed by a scheduler until CPU utilization has declined to a certain threshold. In contrast to the MANE, adaptation decision-taking at the Home-Box has to take more parameters into account, including terminal capabilities and user preferences, but has fewer flows to handle. A Home-Box in a typical household might have to handle up to five concurrent flows. However, any adaptation or transcoding operations have way higher computational complexity and resource demands than the adaptation decision-taking.

Transcoding at the server side and at the Home-Box are computationally expensive parts of SVC tunneling. Transcoding to SVC on the server-side has only to be performed once per video and can be performed offline prior to streaming. Transcoding from SVC to other formats on the Home-Box demands less resources but the Home-Box has to be dimensioned to support a handful of concurrent flows.

In-network adaptation in MST mode relies on receiverdriven layered multicast, thus, the usage of SVC does not put any overhead on this approach. In SST mode, RTP depacketization and re-packetization limit the number of concurrent flows. A prototype implementation on an off-theshelf WiFi router supported concurrent adaptation of several flows in 2008 [13], dedicated hardware and improved algorithms may lead to a higher number of possible concurrent flows.

\section{G. The ALICANTE Adaptation Framework Key Innovations}

The corresponding key innovations are summarized as follows:

- Better network resource utilization based on adaptation and maintaining a satisfactory QoE/QoS: Content is encoded in or transcoded to scalable media formats such as SVC for efficient layered multicast distribution enabling in-network adaptation. End users and network devices provide QoE/QoS feedback to the ADTF, to adjust the service in a distributed and dynamic way.

- Context information from multiple receivers is aggregated at MANEs and used for local adaptation decision-taking. Additionally, adaptation decisions are 
TABLE I.

Y-PSNR RESULTS OF SVC LAYERS FOR THE HALL MONITOR SEQUENCE WITH VARIOUS ENCODERS AND RATE CONTROL MODES

\begin{tabular}{|c|c|c|c|c|c|c|c|c|c|c|c|c|c|c|c|}
\hline \multirow{2}{*}{ Target Quality } & \multicolumn{5}{|c|}{ bSoft (VBR) } & \multicolumn{5}{|c|}{ MainConcept (VBR) } & \multicolumn{5}{|c|}{ MainConcept (CBR) } \\
\hline & bitrate [kbps] & $L 3[d B]$ & $L 2$ [dB] & $L 1[d B]$ & $L O[d B]$ & bitrate [kbps] & $L 3$ [dB] & $L 2[d B]$ & $L 1[d B]$ & $L 0[d B]$ & bitrate [kbps] & $L 3[d B]$ & $L 2[d B]$ & $L 1[d B]$ & $L O[d B]$ \\
\hline$Q 1$ & 4482 & 44.74 & 33.05 & 26.89 & 23.20 & 3270 & 42.97 & 39.15 & 35.94 & 32.96 & 3095 & 43.87 & 42.64 & 41.03 & 37.74 \\
\hline$Q 2$ & 2446 & 42.03 & 32.95 & 26.89 & 23.20 & 1867 & 40.10 & 36.62 & 33.38 & 30.39 & 2202 & 42.30 & 41.07 & 39.53 & 36.30 \\
\hline$Q 3$ & 1244 & 39.84 & 32.77 & 26.87 & 23.21 & 1191 & 37.79 & 34.22 & 30.88 & 27.84 & 1622 & 40.96 & 39.75 & 38.32 & 35.09 \\
\hline Q4 & 699 & 37.83 & 32.48 & 26.86 & 23.23 & 816 & 35.46 & 31.74 & 28.34 & 25.30 & 1058 & 38.68 & 37.43 & 36.03 & 32.62 \\
\hline
\end{tabular}

propagated within the media delivery network enabling distributed adaptation decision-taking. For example, upstream to the server in case of RTP-like streaming or downstream to the receiver in case of HTTP-like streaming.

- Distributed coordination for optimal adaptation and improved bandwidth usage involves the active participation of multiple entities across the media delivery network such as adaptation decision-taking, actual adaptation, and QoE/QoS probes.

\section{TARGETED RESEARCH OUTCOMES AND TEST-BED}

\section{A. Targeted Research Outcomes}

Based on the ALICANTE architecture and the aforementioned key innovations, we target the following research outcomes:

- Guidelines for scalable media encoding/transcoding parameters (with SVC as example) for a given set of use cases enabling in-network adaptation. They will answer questions like how many SVC layers at which bitrate, framerate, and SNR are the optimal choice for the use cases developed in ALICANTE.

- Guidelines for the distributed adaptation decisiontaking framework which basically provides answers to the open questions raised in Section I, i.e., where to adapt, when to adapt, and how (often) to adapt.

- Enhancement of the decision-taking algorithm through the exploitation of the active and passive monitoring framework based on flow- and content-awareness at MANEs.

- Enhancement of the SVC adaptation based on network load/conditions and QoS constraints using a contentaware approach.

- Assessment of the performance and scalability (e.g., number of flows, flow traffic profile) of the developed in-network adaptation mechanisms, in terms of computing resources utilized (e.g., CPU and memory) as well as network related metrics (e.g., processing delay per flow, maximum achieved bandwidth).

- Based on our test-bed and pilots we will determine the (end-to-end) delay (taking real-time applications into account), minimum quality degradation (measured via objective methods, i.e., PSNR, and subjective methods, i.e., MOS), and scalability w.r.t. the number of parallel sessions which should not increase exponentially.

- Mappings of network and device monitoring parameters that enable the prediction of the QoE and the validation thereof through subjective quality assessments.

- $\quad$ A holistic approach for in-network adaptation applying different adaptation policies per content-aware virtual network.

\section{B. Proposed Test-bed Setup}

The proposed test-bed setup for our evaluations is described below and depicted in Fig. 2. The content is encoded or transcoded into SVC by the Encoder/Streamer module. A Traffic Generator creates cross-traffic that is used for the evaluation of DiffServ and adaptation functionalities of MANEs. The mixed traffic is ingested through a MANE into the core network and is simultaneously captured by a Network Traffic Analysis tool. That ingress MANE performs dynamic SVC adaptation and content-aware forwarding over labelswitched paths (LSPs). The traffic is routed through the network via Label Switching Routers (LSRs). At the egress point of the network, we capture the traffic again for network traffic analysis. The SVC content is decoded or transcoded to a suitable terminal format by the Decoder module. The Encoder/Streamer module and the Decoder module correspond to HBs in the ALICANTE architecture and also form the ingress and egress points for SVC tunneling. Finally, the received content is analyzed by the Video Analysis module. The video quality analysis comprises objective methods and a QoS-QoE mapping model [14]. This mapping will be validated using subjective tests.

This test-bed integrates our previous setups of [14], [15], and [16]

\section{SCIENTIFIC RESUltS ACHIEVED So FAR...}

The research challenges and open issues including a description of use cases in question are further detailed in [14]. In this section we summarize scientific results and present test results for comparing rate control modes for SVC tunneling.

\section{A. Achieved Results}

The quality impact of SVC tunneling is investigated in [15] using MPEG-2 as starting point and providing a baseline for further research. The results indicated a total decrease in luminance-component PSNR (Y-PSNR) of $2.1 \mathrm{~dB}$, with around 
TABLE II. BJONTEGAARD DELTA FOR SVC TUNNELING.

\begin{tabular}{|c|c|c|c|c|c|c|}
\hline \multirow[b]{2}{*}{ Sequence } & \multicolumn{2}{|c|}{ bSoft (VBR) } & \multicolumn{2}{|c|}{$\begin{array}{l}\text { MainConcept } \\
\text { (VBR) }\end{array}$} & \multicolumn{2}{|c|}{$\begin{array}{l}\text { MainConcept } \\
\text { (CBR) }\end{array}$} \\
\hline & $\begin{array}{c}\text { BD- } \\
\text { PSNR } \\
{[d B]}\end{array}$ & $\begin{array}{c}\text { BD- } \\
\text { bitrate } \\
{[\%]} \\
\end{array}$ & $\begin{array}{c}B D- \\
P S N R \\
{[d B]} \\
\end{array}$ & $\begin{array}{c}\text { BD- } \\
\text { bitrate } \\
{[\%]}\end{array}$ & $\begin{array}{c}B D- \\
P S N R \\
{[d B]} \\
\end{array}$ & $\begin{array}{c}\text { BD- } \\
\text { bitrate } \\
{[\%]}\end{array}$ \\
\hline fore & -2.08 & 50.3 & -2.03 & 53.7 & -2.40 & 61.6 \\
\hline container & -1.57 & 38.2 & -1.99 & 51.0 & -2.91 & 66.9 \\
\hline $\begin{array}{l}\text { hall_monit } \\
\text { or }\end{array}$ & $-\mathrm{c}$ & 22.6 & -1.40 & 54.1 & -1.82 & 73.6 \\
\hline stefan & -2.59 & 0 & -2.09 & 32.1 & -2.88 & 53.4 \\
\hline Average & -1.74 & 38.04 & -1.88 & 47.7 & -2.50 & 63.9 \\
\hline
\end{tabular}

$1 / 3$ of the quality impact attributed to the initial MPEG-2-toSVC transcoding step. A bitrate increase of $43 \%$ is required to compensate the quality loss which is still less than the necessary bandwidth for MPEG-2 simulcast-based streaming. Based on the test-bed setup described in [15], we performed further tests for comparing the impact of rate control modes for SVC tunneling as detailed later on.

An initial performance evaluation of SVC streaming and real-time in-network adaptation is reported in [16]. Further evaluations with enabled end-to-end QoS control are presented in [14] including a model for QoS-QoE mapping. The video streaming system introduces a cross-layer QoS mapping based framework for media- and user/terminal-aware transmission and management. The experimental results indicate the advantage of such an adaptation system that facilitates the control of bandwidth utilization to obtain an improved perceived video quality for end users.

Finally, initial subjective quality assessments for an application that may benefit from ALICANTE's media ecosystem are described in [17]. In the future it is anticipated to apply such subjective tests on the work conducted in [14], [15], and [16] respectively. Furthermore, we are currently working on combining [15] with the QoS-QoE mapping model of [14] and the deployment of multi-video rate allocation for SVC tunneling.

\section{B. Comparing rate control modes for SVC Tunneling}

We extended the aforementioned tests of [15] for comparing SVC tunneling of variable bitrate (VBR) encoding mode against constant bitrate (CBR) encoding mode using the following setup. The test was performed with the test sequences foreman, container, hall monitor, and stefan, each having a resolution of $352 \times 288$ and $25 \mathrm{fps}$ framerate. The test sequences were initially encoded to MPEG-2, transcoded in a first transcoding step to SVC using pixel-domain transcoding (PDT), and in a second transcoding step back to MPEG-2 using PDT. These transcoding scenarios were performed for VBR and CBR encodings separately. For comparing the required bandwidth of SVC tunneling with MPEG-2 simulcast, we selected for each extracted SVC layer an MPEG-2 encoding with best matching Y-PSNR.

One challenge in this setup is the selection of a suitable quantization parameter (QP) or target bitrate for the SVC
TABLE III COMPARISON OF REOUIRED BANDWIDTHS FOR SVC TUNNELING VS. MPEG-2 SIMULCAST.

\begin{tabular}{|c|c|c|c|c|c|c|}
\hline \multirow[b]{2}{*}{$\begin{array}{l}\text { Target } \\
\text { Quality }\end{array}$} & \multicolumn{2}{|c|}{ bSoft (VBR) } & \multicolumn{2}{|c|}{$\begin{array}{l}\text { MainConcept } \\
\text { (VBR) }\end{array}$} & \multicolumn{2}{|c|}{$\begin{array}{l}\text { MainConcept } \\
\text { (CBR) }\end{array}$} \\
\hline & $\begin{array}{c}\text { SVC } \\
\text { tunneling } \\
\text { [kbps] }\end{array}$ & $\begin{array}{c}\text { MPEG-2 } \\
\text { simulcast } \\
\text { [kbps] }\end{array}$ & $\begin{array}{c}\text { SVC } \\
\text { tunneling } \\
\text { [kbps] }\end{array}$ & $\begin{array}{c}\text { MPEG-2 } \\
\text { simulcast } \\
\text { [kbps] }\end{array}$ & $\begin{array}{c}\text { SVC } \\
\text { tunneling } \\
\text { [kbps] }\end{array}$ & $\begin{array}{c}\text { MPEG-2 } \\
\text { simulcast } \\
\text { [kbps] }\end{array}$ \\
\hline$Q 1$ & 5333 & 3041 & 3694 & 3454 & 3286 & 4721 \\
\hline$Q 2$ & 3446 & 2025 & 2418 & 2082 & 2242 & 3191 \\
\hline$Q 3$ & 2201 & 1452 & 1650 & 1277 & 1687 & 2093 \\
\hline$Q 4$ & 1438 & 1102 & 1132 & 900 & 1109 & 1287 \\
\hline Average & 3105 & 1905 & 2224 & 1928 & 2081 & 2823 \\
\hline
\end{tabular}

encoding in the first transcoding step. In [15], the same target bitrate was used for the initial MPEG-2 encoding and the SVC encoding. Such configuration is not flexible when it comes to saving network resources in streaming scenarios. Thus, we chose an experimental approach were the original sequence is encoded to SVC with several target qualities (i.e., QP or target bitrate) and then the configuration that yields a Y-PSNR just above that of the MPEG-2 encoding is selected. For the second transcoding step (back to MPEG-2), we applied again the target quality of the initial MPEG-2 encoding.

The SVC encoding was configured with four mediumgrained scalability (MGS) layers. We tested two industry-grade SVC encoders, i.e., MainConcept v1.5 [18] and bSoft v120403 [19]. MPEG-2 encoding was performed via FFmpeg v0.8 [20]. The bSoft encoder distributes transform coefficients to create MGS enhancement layers. On the other hand, the MainConcept encoder performs re-quantization to obtain those MGS layers. Compared to the highest layer, we reduced the QP by 6 per MGS layer for VBR or conversely the target bitrate by $30 \%$ for CBR. Since the bSoft encoder always yielded better ratedistortion (RD) performance for VBR mode, we did not perform CBR mode tests for the bSoft encoder.

The starting points of the test are four SVC encoding configurations $(Q 1, Q 2, Q 3, Q 4)$ with highest layer QP of $\{28$, $24,20,16\}$ for VBR and target bitrate of $\{1,1.5,2,3\} \mathrm{Mbps}$ for CBR. The qualities of the SVC layers (labeled $L 3$ for highest layer and $L 2, L 1, L O$ for the lower layers respectively) of the hall_monitor sequence are exemplarily shown in Table 1. While the bSoft encoder yields good overall RD performance, the automatic distribution of transform coefficients allocates little quality to the lower layers (due to a uniform rate distribution among layers) compared to our configuration of the MainConcept encoder.

The Bjontegaard Delta (BD) [21] results for the two transcoding steps are shown in Table 2. The $\mathrm{BD}$ is measured between the initial and final MPEG-2 encoding. As mentioned before, we applied a flexible approach for the target quality of SVC encoding. Thus, the BD is applicable neither to the MPEG-2-to-SVC transcoding step nor the SVC-to-MPEG-2 transcoding step, but only to the result of the entire transcoding chain.

Sequences with lower spatial detail and lower amount of movement (such as hall_monitor, container) typically show 
less quality degradation than those with higher amounts. The overall results show lower quality impact for VBR mode $(-1.74 \mathrm{~dB}$ for bSoft encoder, $-1.88 \mathrm{~dB}$ for MainConcept encoder on average) than for CBR mode ( $-2.50 \mathrm{~dB}$ on average).

Table 3 presents the comparison of average required bandwidths for SVC tunneling and MPEG-2 simulcast streaming. Columns labeled SVC tunneling show required bandwidths for delivering the content which has been transcoded from MPEG-2 to SVC (i.e., first transcoding step). For the second transcoding step, the content is transcoded back into the final MPEG-2 encoding. The required bandwidths for MPEG-2 simulcast (of the initial MPEG-2 encoding) with the same quality (in terms of Y-PSNR) as that final MPEG-2 encoding are shown in columns labeled MPEG-2 simulcast.

Only CBR mode yields lower overall bandwidth requirements for full SVC tunneling than for equivalent MPEG-2 simulcast, reducing the required bandwidth by up to $32 \%$ (and $26 \%$ on average). For the tested configurations, SVC tunneling with VBR mode performs worse than equivalent MPEG-2 simulcast, even though it yields less quality degradation. This is attributed to the comparatively high quality of lower SVC layers in CBR mode (cf. Table 1), which manifests in higher bitrates of MPEG-2 simulcast in order to match that quality. We argue that the bandwidth efficiency of SVC tunneling depends on the configuration of lower SVC layers rather than on the encoder implementation. Furthermore, the number of SVC enhancement layers (ELs) plays an important role for the bandwidth efficiency of SVC tunneling. Future work will investigate whether the additional MPEG-2 encodings for simulcast outweigh the SVC coding overhead of additional ELs in terms of required bandwidth. Note that SVC tunneling with VBR mode may still be favorable to MPEG-2 simulcast in scenarios where only one of the two transcoding steps is needed (e.g., if the client's media player supports SVC), since every transcoding step has an impact on video quality.

\section{CONCLUSIONS}

In this paper we have presented research challenges and key innovations for edge and in-network adaptation featuring a distributed ADTF and SVC tunneling. Towards targeted research outcomes in the FP7 ALICANTE project, we proposed an integrated test-bed. Besides presenting a selection of scientific results achieved so far, this paper provides further results in SVC tunneling with focus on comparing the impact of VBR and CBR encoding modes with respect to quality degradation and bandwidth efficiency. The results indicate smaller quality impact for VBR mode $(-1.74 \mathrm{~dB}$ and $-1.88 \mathrm{~dB}$, depending on the encoder) than for $\operatorname{CBR}(-2.50 \mathrm{~dB})$, but the comparison of required bandwidth only yields bandwidth reduction (of 26\%) for SVC tunneling with CBR mode.

Future work will target high-definition content, subjective tests, and integrating the QoS-QoE mapping of [14] and multivideo rate allocation into the proposed test-bed.

\section{ACKNOWLEDGMENT}

This work was supported in part by the EC in the context of the ALICANTE project (FP7-ICT-248652). The authors thank bSoft Ltd. and MainConcept $\mathrm{GmbH}$ for providing their encoders.

\section{REFERENCES}

[1] R. Mohan, J. R. Smith, and C.-S. Li, "Adapting multimedia Internet content for universal access," IEEE Transactions on Multimedia, vol.1, no.1, pp.104-114, Mar. 1999.

[2] F. Pereira and I. Burnett, "Universal multimedia experiences for tomorrow," IEEE Signal Processing Magazine, vol.20, no.2, pp. 63- 73, Mar. 2003.

[3] H. Schwarz, D. Marpe, and T. Wiegand, "Overview of the Scalable Video Coding Extension of the H.264/AVC Standard," IEEE Transactions on CSVT, vol.17, no.9, pp.1103-1120, Sept. 2007

[4] ALICANTE web site, http://ict-alicante.eu/. Accessed 18 June 2012.

[5] E. Borcoci, D. Negru, and C. Timmerer, "A Novel Architecture for Multimedia Distribution Based on Content-Aware Networking," Proc. CTRQ'10, Athens/Glyfada, Greece, June 2010.

[6] ENVISION web site, http://envision-project.org/. Accessed 18 June 2012.

[7] European R\&D Projects: DANAE, web site, http://cordis.europa.eu/projects/rcn/71233_en.html. Accessed 18 June 2012.

[8] ENTHRONE web site, http://enthrone.org/. Accessed 18 June 2012.

[9] S. McCanne, V. Jacobson, and M. Vetterli, "Receiver-driven layered multicast," Proc. of SIGCOMM'96, pp.117-130, Aug. 1996.

[10] S. Wenger, Y.-K. Wang, and T. Shierl, "Transport and Signaling of SVC in IP Networks," IEEE Transactions on Circuits and Systems for Video Technology, vol. 17, no. 9, pp. 1164-1173, Sept. 2007.

[11] S. Devillers, C. Timmerer, J. Heuer, and H. Hellwagner, "Bitstream syntax description-based adaptation in streaming and constrained environments," IEEE Transactions on Multimedia, vol.7, no.3, pp. 463470, June 2005.

[12] J. Chakareski, "In-Network Packet Scheduling and Rate Allocation: A Content Delivery Perspective," IEEE Transactions on Multimedia, vol.13, no.5, pp.1092-1102, Oct. 2011.

[13] I. Kofler, M. Prangl, R. Kuschnig, and H. Hellwagner, "An H.264/SVCbased adaptation proxy on a WiFi router," Proc. NOSSDAV'08, Braunschweig, Germany, 2008.

[14] B. Shao et al., "An Adaptive System for Real-Time Scalable Video Streaming with End-to-End QoS Control," Proc. WIAMIS'10, Desenzano Del Garda, Italy, Apr. 2010.

[15] M. Grafl, C. Timmerer, and H. Hellwagner, "Quality Impact of Scalable Video Coding Tunneling for Media-Aware Content Delivery," Proc. ICME'11, Barcelona, Spain, July 2011.

[16] N. Zotos, G. Xilouris, D. Renzi, B. Shao, and A. Kourits, "Performance evaluation of $\mathrm{H} 264 / \mathrm{SVC}$ streaming system featuring real-time innetwork adaptation," Proc. IWQoS'11, San Jose, California, June 2011.

[17] M. Waltl, C. Timmerer, and H. Hellwagner, "Improving the Quality of Multimedia Experience through Sensory Effects," Proc. QoMEX'10, Trondheim, Norway, June 2010.

[18] MainConcept web site, http://mainconcept.com/. Accessed 09 May 2012.

[19] bSoft web site, http://bsoft.net/. Accessed 09 May 2012.

[20] FFmpeg web site, http://ffmpeg.org/. Accessed 09 May 2012.

[21] G. Bjontegaard, "Improvements of the BD-PSNR model," ITU-T Q.6/SG 16 Video Coding Experts Group, doc. VCEG-AI11, Berlin, Germany, 2008. 